\title{
Imaging in Oncological Urology
}

J.J. de la Rosette, M.J. Manyak, M.G. Harisinghani, and H. Wijkstra, eds.

London, U.K.: Springer, 2009, 443 pages, $\$ 169$

This book provides an excellent review of the most common tumors in urologic oncology, that is, adrenal and renal cell carcinomas; upper and lower urinary tract urothelial cell carcinomas; and prostate, testicular, and penile carcinomas. Multiple international editors and international chapter collaborators representing radiologic and clinical authorities contributed.

The book is divided into 8 parts with 42 chapters. A novel and consistent format for each chapter is presented. Each is subdivided into the following sections: an introduction, presented by a urologist; conventional imaging techniques, cross-sectional imaging techniques, and radionuclide imaging, presented mostly by radiologists; and "Considerations," a description of the impact of imaging technology on the practice of urologic oncology, presented by a urologist. Parts I and II describe adrenal and renal cell carcinomas, respectively. Urothelial cell carcinomas in the upper and lower urinary tracts are presented in parts III and IV. Part V addresses prostate carcinoma, and parts VI and VII are dedicated to testicular and penile carcinomas. The last part, VIII, addresses the future directions of imaging (sonographic, virtual, optical, elastographic, molecular), gene/drug delivery, contrast agents, and robotics.
The intended audience is radiologists, urologists, and their trainees, but other health care providers who can benefit include nuclear medicine physicians, urologic oncologists, radiation therapists, physician assistants, nurses, and the medical directors of third-party payers. This is a highly recommended book that should be in the reference libraries of these health care providers and in medical libraries that service health care providers. The book is concise, consistent, and easy to read, and it presents state-of-the-art imaging in urologic oncology. The images, diagrams, figures, and references are excellent.

The image-guided therapeutic interventions, decreased morbidity, improved outcomes, and decreased costs described in this book will greatly benefit patients. The multidisciplinary approach to urologic cancer management demonstrated by this book makes it a great contribution to the medical literature, and the authors are to be highly commended for their efforts.

Aurelio Matamoros, Jr.

M.D. Anderson Cancer Center

1515 Holcombe Blvd., Unit 1273

Houston, TX 77030

E-mail: amatamor@di.mdacc.tmc.edu

COPYRIGHT ๑ 2010 by the Society of Nuclear Medicine, Inc.

DOI: 10.2967/jnumed.110.075515 